\title{
Educators' Perspectives on Environmental Education in India
}

\author{
A Case Study in School and Informal Education Settings
}

\author{
Haein Shin and Srinivas Akula
}

\begin{abstract}
This chapter documents the perspective of educators on the ability to teach environmental education (EE) in formal and non-formal education settings. It aims to answer the question "How should educators be prepared so that $\mathrm{EE}$ is effective and meaningful for the learner?"

Based on interviews with a school principal, a schoolteacher, and facilitators at two community vocational and life-long learning centers, the chapter highlights perspectives on EE, training and implementation challenges, and successes in integrating EE into overall learning and training programs.

We discuss the existing fragmented approach to EE; although it increases environmental awareness and activities overall, the increase comes with only limited understanding of the depth and linkages of environmental issues. Three approaches show promise in improving current $\mathrm{E}$ E delivery: offering teacher training that conveys more environmental knowledge, especially in relation to global and local carbon footprints; giving systemic priority to $\mathrm{E} \mathrm{E}$ and offering incentives to teach it; and engaging learners through inquiry-based learning.
\end{abstract}

\section{Keywords}

teacher development - teacher support - environmental education - communitybased learning

Texts reflecting India's cultural notion of humans' oneness with the universe and with nature span millennia, from thousands of years back all the way up to the 1950s Constitution of India, which decrees that harmony with nature and 
its protection are fundamental duties of Indian citizens (Almeida, 2011). This interconnectedness, both cultural and in reality, presents a paradox. India's sheer size positions it as a critical player in the global environmental landscape. It is home to more than 1.3 billion people and ranks as one of the fastest growing economies in the world (ВвС News, 2019). It is also the third largest carbon dioxide emitter in the world, after China and the United States (Global Carbon Atlas, 2018). The magnitude of the pollution it generates thus stands at odds with its deep-rooted cultural respect for nature.

The cultural underpinning is manifest in the education system, at least at the level of policy, where the country has shown commitment. It prioritized environmental education (EE) in a 1991 Supreme Court mandate. And subsequently, in 2003, the government mandated that the National Council of Educational Research and Training (NCERT) produce extensive content on EE (UNESCO, 2016). As a result, more than 300 million students in 1.3 million schools had received some form of EE training as of 2015 (UNESCO, 2016).

Even though India gives EE certain cultural and policy priority, the quality of its implementation, teaching, and effectiveness needs to be better understood. This chapter's argument is framed on the article "Roadblocks to Quality Education in a Time of Climate Change" (Kwauk, 2020), one of the five roadblocks being limited teacher capacity and teacher support on EE. Certainly, public attention to $\mathrm{EE}$ and its contents and implementation have surged - they have even spread into non-profit and non-governmental organizations globally - but "teachers lack the systemic support to become change agents for sustainability" (p. 15). Our aim in thinking about EE in India was to answer the question "How should educators be prepared so that EE is effective and meaningful for the learner?" We sought to answer it by getting the perspective of educators.

This chapter first outlines India's EE-specific policies on teacher training and capacity building as to curriculum and pedagogy. It highlights findings from the literature on challenges in these areas. Against this backdrop, we present themes that emerged from interviews with educators who have direct experience in teaching and managing EE. Their cross-sectional perspectives are followed by discussion, analysis, and further considerations concerning teacher education and systemic improvements.

\section{$2 \quad$ India's Curricular and Pedagogical E E Framework}

At the policy level, India's curricular and pedagogical EE framework aligns with the integrated learning approach promoted in education for sustainable 
development (ESD) globally and also with the UN's multi-pronged economic-social-environmental pillars that underpin its Sustainable Development Goals (United Nations, 2020). India's curricular framework echoes the latter's emphasis on education that encompasses knowledge and skills that refer to, and relate to, the interconnectedness of issues in the world.

India's guide called Teachers' Handbook on Environmental Education for Classes XI-XII articulates such an approach: "To ensure the continuation of proactive action towards the environment ... the core focuses on the interconnected nature of the physical-biological-social-economic system pertinent to environmental issues" (NCERT, 2011, p. XI). Although the guide recommends that teachers explore individual and group project-based learning, it puts EE in the science and the social studies syllabi rather than making it a course on its own.

NCERT's National Curriculum Framework for School Education includes the environment at all levels. At the primary stage, teachers are to encourage learners to foster a culture of awareness and interest via "concrete situations related to immediate environment", then lead to more structured environmental study activities. At the upper primary stage, learners are to understand the nature of the world and its changes, with emphasis on natural resources in local and global contexts, along with topical issues of water, health, nutrition, and family welfare, among others. At the secondary stage, teachers are to help learners experiment and conduct activities in and out of school, including activities with industries (farming, factories) and community.

One foundational issue in teacher education is how curricular and pedagogical frameworks and recommendations on paper are not fully realized in actual teacher education, and this issue applies in EE. Dhull and Verma (2017), analyzing the question in India, cited similar challenges across the globe in building teacher capacity. Teksoz et al., writing in 2010 (as cited in Dhull \& Verma, 2017), found that most pre-service teachers agreed that EE should be mandatory and that every teacher should be environmentally literate. However, teachers felt inadequately prepared for integrating emerging environmental issues into their courses, primarily because they lacked knowledge and skills and had no in-service trainings guiding them. In another study reported by Molosiva in 2010 (as cited in Dhull \& Verma, 2017), teachers commenting on lack of resources said that education authorities often assumed teacher capacity when, in fact, teachers themselves felt unprepared.

In addition to problems with insufficient teacher training in understanding environmental issues, the problems with EE extend into teaching methods. Several studies documented lecture style as the most common method teachers used to teach EE. Teachers thought it the only suitable approach for when 
large audiences are involved. Hasan and Ismail wrote in 2011 (as cited in Dhull \& Verma, 2017) that innovative methods or outdoor learning activities were not practiced. In fact, given teacher shortages, using organized outdoor space for hands-on EE, inquiry-based learning, may pose a significant challenge. Although it is reported that there are about 32 pupils per teacher in India's primary education (Statista, 2017), teacher shortages and teacher absenteeism likely affect the ratios in reality. Dhanalakshmi and Kumar (2012) in studying rural and urban schools in the area of Bangalore, found that respondents $(78 \%$ of urban and $62 \%$ of rural) felt that teacher absenteeism was great enough to influence the quality of education.

Another systemic challenge is that teachers are deployed across the country to teach in rural and urban schools in communities that they may not be from. Teacher education, support, and resources are thus dependent on what is available on-site. Further, even when teachers do try to convey content knowledge, meet expectations, and use place-based pedagogies and local examples for fuller learning, they are also, as newly placed teachers in new work sites, "expected to become quickly rooted in communities in which they are outsiders. Such a student-centered focus - while important in the classroom - means that their needs as new teachers to build their professional resilience, adaptive capacity, and sense of place and community are ignored" (Kwauk, 2020, p. 17).

Against the backdrop of EE policy overlaid with the challenges in teacher education and implementation, the following sections examine educators' own perspectives and voices.

\section{3}

\section{Methodology}

What provided the initial impetus for this chapter was an effect the authors observed: a suggestion to include EE topics in the curriculum of an Information and Communications Technology (ICT) Center in Mahbubnagar, Telangana State, led to active EE sessions in the overall training program. This effect prompted our inquiry into how educators understand $\mathrm{EE}$, what challenges exist both in and out of formal education systems, and what shows promise in implementing EE.

The ICT Center, a community-based center, powered by solar energy, trains women and youth in digital literacy, job readiness, soft skills, and life skills. Partners in this program include the district government (District Magistrate); the Center for Sustainable Development (CSD) of the Earth Institute at Columbia University; and Infrastructure for Sustainable Development (i4SD), which designs, and promotes affordable access, to infrastructure (energy, water, 
transport, and connectivity). The education team from CSD provided the initial curriculum framework, contents, and advising, and from there the facilitators of each ICT Center supplied the initiative to teach EE. The curriculum of the first ICT Center in Mahabubnagar, informed the work of another ICT Center's training program in Bhopal, Madhya Pradesh.

We interviewed the facilitators in both centers to understand how EE has taken shape in their respective institutions. In those interviews, references to the local formal school systems came up; therefore, we added interviews with a local public school principal and a local public school teacher. We could then document a cross-sectional case study, that is, explore how EE is experienced, by educators in community-based informal learning centers and in a formal school system. Because we sought to examine teacher capacity in EE, we used targeted sampling so as to select interviewees who were already implementing some level of $\mathrm{EE}$ in their classrooms.

\subsection{Agreement That EE Is Important But Varying Views on What EE Is}

We need to examine the actual implementation of $\mathrm{EE}$, but first the question we are trying to answer calls for an even more basic sense of the educators' own understanding of the definition and scope of EE. When asked what they saw as the core principles and content, all four educators shared the view that $\mathrm{EE}$ is about human interaction with the environment and that human activity has implications for nature. In all conversations, we encountered the cultural underpinning of respect for nature, in which Mother Nature is seen as the entity that shields the world and provides for it - supplying oxygen, food, safety, and protection. All the educators expressed a sense of responsibility and care for future generations, which bolsters the argument for encouraging EE.

Despite their shared understanding, they demonstrated less clarity and unity on details of the scope and content of EE. One educator described EE as "a kind of education which will seek to make pupils fully aware of the problems connected with their environment so that they will be able to tackle these problems with a sense of responsibility and with the technical skills which will enable them to contribute to their solutions along with other members of their community". The school principal saw prevention of harm to the environment as the focus, whereas the schoolteacher viewed EE as a process of progressing from awareness to action. The teacher said of $\mathrm{EE}$ that it is a way "to promote the awareness and understanding of the environment, its relationship with man and his activities. It is also aimed at developing responsible actions necessary 
for preservation, conservation, and improvement of the environment and its components". The teacher also believed that any curriculum promoting environment-friendly objectives should be considered an EE curriculum.

The two facilitators in community-based centers referenced more specific environmental issues in their locales. Even though they teach in different states, they both approached EE through a more topic-based lens, noting the need for specific awareness regarding burning garbage, personal and environmental hygiene, plastic pollution, air pollution linked to global warming, and planting of trees, with the ultimate goal of doing as much as possible to avoid the environmental disasters in the world.

Thus, although the cultural premise of oneness with nature resonates among all educators, their focuses vary. Despite the good EE intentions of all, the diversity in their definitions of it illustrates one of the roadblocks to systemically tackling $\mathrm{EE}$, namely, that even in the same town, few educators have internalized the same aspects of $\mathrm{EE}$ as their guiding concept. The responses of the educators reveal a common language as to a framework for $\mathrm{EE}$ on one hand and varying and less defined emphases as to detailed particulars on the other hand. This finding suggests that educators in general may also have exposure to broad EE policies and topics but lack guidance as to a defined scope and detailed implementation. Such a state of affairs can have the negative effect of essentially leaving educators to take on $\mathrm{EE}$ on their own. Lack of coordination can lead to a fragmented delivery of EE content.

\subsection{The Government's Environment Initiatives Influence the Content of Teacher Training}

To understand how the educators' perspectives on EE translate into their actual teaching, we asked them what they hope their students will learn. All mentioned specific topics that can lead to a better environmental outcome: pollution; tree planting; waste management; and personal and environmental cleanliness and hygiene. These topics are action-oriented and doable at both individual and school levels. But emphasis on this list also suggests that the primary focus is treating the symptoms of environmental degradation as opposed to addressing the root problems of sustainability and the imbalance between human activity and the needs of nature.

The list also turns out to mirror that of the government's initiatives on the environment. Educators in both the community-based and formal education settings were familiar with the district-level activities based on their adoption of programs related to India's Open Defecation Free (ODF) status. In the Mahabubnagar District, the adoption of 2014's national program involved the following aspects: 
- Water, sanitation, hygiene (WASH);

- Swachh Bharat-Swachh Vidyalaya: "personal hygiene, grooming, school cleanliness";

- Menstrual hygiene management;

- Nurseries and tree planting;

- Construction of toilets;

- Waste management;

- Plastic ban.

These initiatives have scaled up at the district and community levels, with awareness campaigns, door-to-door campaigns, training in producing reusable materials, distribution of reusable materials, inclusion of women in initiatives, educational programs, tree planting, collective clean-ups, and improvements to infrastructure.

The national and sub-government-level programs seem to have been effective in creating overall awareness in the educators both in and out of formal schools, specifically in terms of improving hygiene and cleanliness in the immediate environment. However, the government initiatives are still significantly limited in terms of giving insight into the root causes of broader sustainability issues, including an understanding of climate change and carbon footprints.

Further, these initiatives have implications for teacher training. The topics directly guide the scope and content of trainings educators receive on EE. Teacher training thus remains limited to these action-based, not-quite-comprehensive environmental concepts.

\subsection{EE Teacher-Training and Teaching}

Discussions with the educators suggest that awareness of $\mathrm{EE}$ is becoming more widespread, but even with local government initiatives and school-community activities, only some of this awareness translates into actual conveying of educational content. This lack seems to stem from a combination of the two challenges mentioned above: (a) trainings of educators are limited in scope, focusing on addressing symptoms of environmental degradation rather than on conceptual linkages and real linkages, and (b) trainings of educators do not explicitly cover how to integrate $\mathrm{EE}$ into a class or teaching event, leaving implementation up to the initiative and willingness of individual teachers.

According to the school principal and schoolteacher, teachers receive halfday Swachh Bharat-Swachh Vidyalaya trainings three times a year at the district office. One phase covers school cleanliness, tree planting, WASH (water, sanitation, hygiene), and waste management; another phase covers personal grooming and hygiene. 
The school principal has seen parent groups, NGOs, and Swachh Bharat teams offer teaching events for young people on the environment, but the focus seems to be on one-off activities. For example, Grades 1 through 10 have school projects for planting trees, cleaning the school grounds, and learning about types of pollution (air, soil, water). The government initiates specific trainings of educators; then teachers and community members take up implementing activities in the school and its surroundings. All educators describe the teaching events as lecture-based but with accompanying activities planned by the district government in collaboration with communities. Action-oriented activities involve the topics mentioned in the preceding section. Critical topics such as climate change are missed.

The government initiatives do have some merit, however, in that they reach a wide population. For example, although the two facilitators in the ICT Centers had no prior training in EE, cross-fertilization between school and community in relation to the above kinds of events has exposed educators in both schools and ICT Centers to similar activities, which then get incorporated as activities into their respective individual teaching. If the same structure for delivering information to the community were employed but with better curated and more holistic EE contents in the teacher trainings, access to quality EE information could attain a wider reach in both schools and community.

More support is needed to achieve effective incentives to teach EE, sustained attention to it, and good content. All four educators find that in the classroom, EE is still treated as a minor syllabus item to get through, not as a rigorous subject that students must learn and internalize. None of the four educators recalled (apart from school-wide one-off activities) any in-depth classroom discussion, any in-class activity, or any classroom explanation tying environmental issues to sustainability problems in the immediate physical environment. In the formal classroom, the environment is covered in a simple presentation of topics.

When the two facilitators were students, they recall, environmental topics were presented in a lecture but not taken seriously by the teacher or the students. The principal noted that EE "depends on the concerned schoolteachers. All the teachers are attending training, but only 40 to $50 \%$ of schools are implementing what has been learned in training". Both the principal and the schoolteacher noted that the environment is not taught as a separate subject. It has no designated, specialized, trained teacher with some expertise. The seemingly robust EE policy descriptions, which detail the importance of EE from primary to tertiary cycles, still put $\mathrm{EE}$ in the science and the social studies syllabi rather than giving it its own course. The implication for teachers is that $\mathrm{EE}$ is less important than other subjects and that it's okay that teachers are 
ill-equipped to teach it. Both attitudes feed the probability that EE topics will get lost or dropped in favor of other classroom lessons.

\subsection{Winning E E Approaches in Informal Classrooms}

In spite of educators' challenges in implementing $\mathrm{EE}$, the experiences at the community ICT Centers show promise with regard to effective EE delivery. The centers have managed to achieve meaningful learner engagement. They take advantage of inquiry and discussion-based learning. Their students enjoy access to information on computers with good connectivity. Their students develop an awareness and appreciation of issues seen in the immediate environment.

In the ICT Center in Mahbubnagar, the facilitator focuses on waste management, tree planting, and plastics, which relate to visible issues and hazards seen daily near the center's grounds. In an effort to link causes of environmental issues and their consequences, the facilitator, on his own initiative, shared information about current events, finding, for example, articles and photos online about the wildfires in Australia and their connection with climate conditions. Not only was online information thus disseminated, but more important, when students learned of environmental issues in the news, the facilitator followed up with group discussions. Trainees were encouraged to do further computer research themselves and to share their findings. They were often asked to create group presentations about their findings, as a form of peer learning.

In the ICT Center in Bhopal, the facilitator's emphasis on plastic pollution links to a local government's Bring-Your-Own-Bag initiative. Partnering with a government program, trainees produced reusable bags from recycled fabric. The trainees witnessed the magnitude of plastic pollution in their midst, received vocational training to produce reusable products, and were given environment lessons by the facilitator. All these factors reinforced in students' minds the importance and value of the products they were producing and helped them understand the root cause in the environment of the problem that their program was helping to alleviate. Compared to a formal classroom, the whole ICт Center set-up (its "learning platform") offers a more holistic lens through which learners can see the systemic cycle linked to an environmental issue: the cause (plastic consumption and usage); the magnitude of the issue (visibly seen in the immediate, local environment); a potential solution (government-supported use of recycled bags at scale); ripple effects and benefits (waste reduction and upcycling of materials and cloth, job training for creation of products, earnings and economic gains from producing recycled products). Even though the full picture of carbon emissions and climate change linkages 
are still missing, such an example of the cause-and-effect, cyclical, and interconnected nature of environmental issues is critical, applicable, and manageable for the trainees.

According to both ICT Center facilitators, their biggest success was students' adopting practices based on what they had learned at the center, instituting recycling in their own homes, for example, and starting to get their friends to do the same. The trainees took what they had discussed in the ICT Center beyond the classroom. And their enthusiasm was evident: they took on additional writing assignments on environment topics and came back to subsequent classes with more questions.

This trainee enthusiasm appears to be new-found. The centers have trainees who also attend local public schools. Both facilitators found these students' basic environmental knowledge to be extremely low before they started learning about the environment at the centers. One facilitator, for example, showed trainees images of various renewable energy sources in use at the ICT Center itself. The trainees recognized and named the various types of renewable energy. But when the facilitator showed them an image of a windmill, they were neither able to state its purpose nor describe how it worked. A sample of four educators is too small for a full assessment of the degree to which students have or have not absorbed information about the environment in their formal schooling, but the overall trend gleaned from 400 trainees in the ICт Centers who also attend formal schools is that the students seem to be learning about the environment only in the ICT Centers. They appear to have no prior knowledge of, or experience with, environmental issues.

Neither ICT Center facilitator received the official EE related trainings that the formal teachers received, but the facilitators seem to be incorporating more extensive EE content into their programs than do the formal-school educators whom the school principal and the schoolteacher talked about. While the names of the topics may be the same (plastic, planting trees, hygiene), the facilitators design their sessions to have greater application to daily life, and they highlight the need to further disseminate environmental information beyond the classroom and into homes and neighborhoods. The group discussions that the facilitators use not only allow trainees to share their existing thoughts and knowledge about environmental problems, but they also allow the group to collectively "ideate" (generate ideas for) potential solutions and to conduct inquiry-based research and group work.

What can also be gleaned from the ICT Center experiences is that although environmental information and resources are important, especially if readily available online, even more important is that the facilitators consistently use the online materials for inquiry-based and participatory learning. 
Facilitators devote some class sessions exclusively to E E topics, which is not always the case at formal schools.

The center's class sizes are about 25, which may permit more individual attention and student engagement than is possible in public schools, with their shortages of teachers.

For formal schools to better manage EE, systemic support and changes will be required. Schools might draw on what the ICT centers know or otherwise conduct teacher training that makes clear environmental linkages and implications for daily life. Necessary changes include mandating separate and dedicated time for $\mathrm{E} \mathrm{E}$, with material and content support, inclusion of critical topics such as climate change and its pervasiveness (clearly explained), and employment of inquiry-based learning methods.

\section{$5 \quad$ Recommendations and Conclusion}

To conclude this chapter, we review and make several recommendations.

A big issue the interviews point out is that regardless of setting, curriculum, culture, or policy, EE still rests on the shoulders of individual educators and relies on the level of their own personal commitment to implementing it.

Even though trainings are currently provided to all teachers in schools, fewer than half of those trained implement $\mathrm{EE}$ in their classrooms, and the E E is limited to action-based improvements that address the symptoms of environmental degradation without any in-depth understanding of core sustainability and climate issues.

Educators thus are left to struggle with what to do in their classrooms. The seven topics outlined in the government policy are the somewhat limited foundation on which educators flesh out their own lessons. The ICT Centers' experience shows promise for an alternative. Facilitators in these community centers are successfully able to engage in fuller and deeper discussions and to apply local examples linking cycles of ecosystems (consumption-upcycling-reuse) to eco-friendly products and behaviors, all with active learner participation.

Our investigation convinced us of the value of the government's giving direct guidance. All four educator had extracted ideas from the content of government-led Swachh Baharat initiatives - and then made their own modifications and implemented them in their classrooms. The government structure holds promise also in disseminating information more widely into communities, beyond the formal schools. We saw evidence that environment topics from government trainings had not only reached and informed teachers but had also been disseminated at the community level. 
Building on the current guidance structure could be valuable for EE training on two levels. First, a national curriculum and national trainings for teachers, with explicit discussion of the systemic and cyclical links between climate change and environmental issues, could fill the current gaps in teacher training. Second, provision through district organization and local schools of examples, content, and materials to teachers could show how EE has been given context and been adapted to local conditions in community settings and other non-national settings, such as vocational training centers like the ICT Center.

Following are discussions of our three recommendations to improve $\mathrm{EE}$, which are (a) to enhance content, (b) to exploit the greater flexibility that non-formal education settings have compared to formal school settings, and (c) to make $\mathrm{EE}$ the priority it deserves to be.

\subsection{Adding the Missing Climate Change Content}

Even though EE in the community centers enjoys some documented success, the magnitude of the pieces that are missing - carbon footprint and climate change - is significant. These topics need more explicit discussion in trainings and in all classrooms. Even when information about pollution and global warming is presented, the coverage is superficial. Missing are key understandings: about the value of renewable energy; the need for policy mandates concerning public transport; the urgent need to pressure businesses, industries, and the market at a system level. Such key content needs strengthening. Also, EE needs to link understanding problems with understanding the behaviors that could constitute solutions. Without such changes, EE will not live up to its potential as a means to effect climate change.

As India's country-wide digitization continues, not only does access to information on EE need to be made a priority, but also the range of material for trainings should be broadened and made more holistic, with emphasis on climate change and carbon footprints.

\subsection{Making Full Use of Lessons from Community-Based Education Settings}

Educators in formal education settings could benefit from exchanging lessons and ideas with community-based educators. A community-based ICT Center, in which the educator has more autonomy to experiment, test, and more readily and quickly adopt new topics and strategies, provides a model for innovation and advancing teaching and learning. Its practices could profitably be further explored.

Our ICT Center facilitators were able to use the infrastructure in their centers (computers, and solar panels that gave a steady supply of electricity) not 
only for learners to gather environmental information, but also and more important, to engage learners in personal research, peer research, inquiry, and investigation of their immediate environments. Formal schools would benefit from these ways of teaching. (It would also help formal schools if their classes were as small, frequent, and fully staffed as those in ICT centers.) These hands-on features, if implemented in formal schools, would move things in the direction of the systemic change required if $\mathrm{EE}$ is to be more firmly established as a school priority.

The fact that IСт Center encourages trainees to discuss and think together on particular environmental topics, and to make full use of digital information, including in groups, fosters teamwork and camaraderie that enable people to adopt sustaining practices together, and even to extend them into their homes and neighborhoods.

\subsection{Making EE an Actual Priority}

Even though EE is labeled a priority in policy and by educators, the absence of academic rigor in $\mathrm{EE}$ and the lack of incentives to focus on it mean that $\mathrm{EE}$ fails any real test as a priority endeavor. It is not treated with the same seriousness as other subjects, which have their assessments, examinations, and evaluations of how they are taught. EE enjoys no such assessments, exams, or feedback.

In schools with teacher shortages, including that of the school principal interviewed, teachers are tasked with teaching multiple subjects. In such a setting, a subject that is not assessed and evaluated will be the first the teacher will forego.

A further element that contributes to the laxness with regard to $\mathrm{EE}$ is some ineffectuality on the part of environmental professionals and environmental enterprises. A Mongabay-India article quotes Mumbai-based environmental activist Rishi Aggarwal (Tandon, 2018), who says that in the 199os at least 100,00o students went through nature education programs run by groups of environmental experts and naturalists. Aggarwal says, "If nature education was successful, we should have had at least a chunk of those students now working in environmental conservation. But that's not the case. Those programs have just produced more eco-tourists" (Tandon, 2018). Aggarwal asks where the environmental leaders are that all those years of environment education should have generated. An educator comments along similar lines:

With the primary focus on dispensing a collection of facts and figures about the environment, and prescribing oversimplified solutions to overgeneralized problems like portraying deforestation as bad and planting 
trees as good, the environmental science education in this country falls short in meeting its ultimate goal - making students really aware of the single biggest issue of our time and [inspiring] them to work towards it. (Narlanka, 2017)

These critics offer a sobering view of the results that can follow from misdirected and unfocused education. Aggarwal believes that elements of activism, governance, and the communication skills necessary to navigate complex and bureaucratic policymaking are all critical missing pieces (Tandon, 2018). A different education system is needed starting now, one that prioritizes these elements and rewards effective teacher trainers and teachers, who are essential to producing well-informed and active learners. At the societal level, environmental research, jobs, and government investment need to co-exist with environmental education in schools, to further motivate and inspire learners to seriously pursue lifelong commitments to the environment and to thorough understandings of this central issue of our time, which has direct implications for human survival and sustenance globally.

\section{References}

Almeida, S., \& Cutter-Mackenzie, A. (2011). The historical, present and future ness of environmental education in India. Australian Journal of Environmental Education, 27(1), 122-133. https://www.jstor.org/stable/44668283

BBC News. (2019, May 31). India loses place as world's fastest-growing economy. https://www.bbc.com/news/business-48478028

Dhanalakshmi, K., \& Kumar, V. C. (2012). An exploratory study on rural and urban schools: A general view. International Journal of Engineering and Management Research, 2(8). https://ijemr.in/wp-content/uploads/2018/o1/AN-EXPLORATORYSTUDY-ON-RURAL-AND-URBAN-SCHOOLS-A-GENERAL-STUDY.pdf

Dhull, P., \& Verma, G. (2017). Environmental education in teacher education and challenges. International Journal of Academic Research and Development, 2(5), 84-87. https://www.researchgate.net/publication/332446189_Environmental_Education_ in_Teacher_Education_and_Challenges

Global Carbon Atlas. (2020). Country emissions: $\mathrm{CO}_{2}$. http://www.globalcarbonatlas.org/ en/CO2-emissions

Kwauk, C. (2020). Roadmaps to quality education in a time of climate change. Brookings. https://www.brookings.edu/research/roadblocks-to-quality-education-in-a-timeof-climate-change/ 
Narlanka, S. R. (2017, October 23). How environmental science in India is failing its students and what to do about it. Research Matters. https://researchmatters.in/ article/how-environmental-science-education-india-failing-its-students-and-whatdo-about-it

NCERT [National Council of Educational Research and Training]. (1992). Environmental education in national policy documents: National policy on education 1986 (With modification undertaken in 1992). Ministry of Human Resource Development. http://www.ncert.nic.in/html/pdf/environment/envstudies/annxı.pdf

NCERT. (2011). Teachers' handbook on environmental education for Classes XI-XII. Department of Education in Science and Mathematics, http://ncert.nic.in/book publishing/environ_edu/handbook/content.pdf

Sharma, V. M. (2010). Environmental pollutions: Ancient solutions in the light of Sanskrit literature. In K. R. Gupta (Ed.), Environmental education in India (p. 169). Atlantic.

Statistica. (2017). Average number of students per teacher in India from 2013 to 2017, by type. https://www.statista.com/statistics/6o3889/pupil-teacher-ratio-in-india-byschool-type/

Tandon, A. (2018, September 18). With 'upcycling labs', 'safai banks' and more, can environmental education save our planet? Citizen Matters. https:/citizenmatters.in/ environment-education-schools-sustainable-development-schools-8213

UNESCO. (2016). Education for people \& planet: Creating sustainable futures for all [Global education monitoring report series]. https://unesdoc.unesco.org/ark:/ 48223/pfoooo245752 